\title{
Waves and Vortices of Rust on the Surface of Corroding Steel
}

\author{
K. Agladze ${ }^{\dagger}$ and O. Steinbock* \\ Department of Chemistry, Florida State University, Tallahassee, Florida 32306-4390
}

Received: June 21, 2000; In Final Form: September 8, 2000

\begin{abstract}
Two-dimensional wave patterns have been observed on corroding steel plates. A thin layer of oxygen-containing nitric acid covering the surface of the low-carbon steel induces the corrosion process. The spectrum of the observed patterns includes propagating pulses, rotating spirals, and serpentine structures. The propagation velocity is on the order of millimeters per second and increases with acidity. Waves can be observed with the naked eye as traveling brownish bands on the passivated grayish steel. Spiral wave tips deposit black lines onto the surface, documenting the tip trajectory and influencing the propagation of subsequent spirals. A simple chemical model for the mechanism of pattern formation is suggested.
\end{abstract}

Excitation waves are known to play an important role in the self-organization of a wide variety of nonlinear media, including biological, chemical, and ecological systems. ${ }^{1,2}$ An important prerequisite for these waves is the existence of local excitable kinetics or relaxational oscillations. ${ }^{1}$ In particular, many electrochemical systems are known to show these regular or chaotic nonlinear oscillations. ${ }^{3}$ They also exhibit complex temporal behavior that cannot be explained in terms of purely local kinetics. ${ }^{3,4}$ Recently, the existence of spatial inhomogeneties was demonstrated for the electrochemical oxidation of formic acid on platinum ring electrodes ${ }^{5}$ and in the anodic dissolution of nickel. ${ }^{6}$ Earlier, several groups reported propagating waves of activation on single steel wires ${ }^{7-9}$ and wire grids submerged in acidic solutions. ${ }^{9}$ However, these studies of spatiotemporal patterns are often hindered by the necessity of using multielectrode arrays ${ }^{5,7}$ for detection. Here, we provide direct evidence for two-dimensional wave patterns on the surface of corroding steel (iron-nitric acid system, or $\mathrm{Fe}-\mathrm{HNO}_{3}$ ). The waves can be observed with the naked eye, and they have a characteristic speed on the order of millimeters per second.

Figure 1 shows an example of propagating waves and rotating spirals on the surface of a low-carbon steel plate. The plate is kept on ice and is covered by a $0.5 \mathrm{~mm}$ thick layer of nitric acid (Fisher, 15.8 N) and water solution (volume ratio 3:1). In the absence of external perturbations, the system is spatially homogeneous and protected by a passivation film which suppresses iron dissolution. The passivation film is a light-gray coating of the steel plate. The dissolution reaction can be initiated locally by breaking the passivation film. In our experiments, initiation was accomplished by touching the surface of the plate with a zinc rod for a short time (about $1 \mathrm{~s}$ ). At the point of contact, a dark, slightly brownish spot appears. Further evolution of this active reaction zone depends on the water content of the solution and on the temperature. These two parameters control the rate of dissolution and the stability of

\footnotetext{
* Corresponding author.
}

$\dagger$ On leave from the Institute of Theoretical and Experimental Biophysics, Pushchino, Moscow Region 142292, Russia. the passivation film. Accordingly, the wave dynamics becomes more active with increasing water content or temperature. In this paper, we will use the term "activity" to describe this phenomenological behavior of the system. We found that for low water content (less than 1:4), the reaction zone survives for a few seconds and then collapses. However, for a volume ratio of water to nitric acid greater than 1:4, traveling waves can be initiated, which start to propagate outward with a characteristic speed of about $0.5 \mathrm{~cm} / \mathrm{s}$. After the first wave has passed through the system, the surface color returned to its grayish state, and it is possible to initiate subsequent waves following the procedure outlined above. This phenomenon differs from what is known as the "iron nerve" system, ${ }^{7-9}$ where local defects of the passivation film propagate with velocities ranging from 5 to $100 \mathrm{~cm} / \mathrm{s}$. Another difference is the striking dark color of the propagating waves (Figure 1), which makes them easy to observe and record. Notice that the existence of these unusually slow waves was already mentioned by Lillie $^{7}$ for the case of steel wires. However, no experimental details were presented.

Traveling waves in the $\mathrm{Fe}-\mathrm{HNO}_{3}$ system are less stable for lower water content. They can break apart, and the evolution of the resulting wave segment depends on the activity of the system. If the activity of system is low, the fragments of the wave front propagate for some distance, usually on the order of $10-15 \mathrm{~mm}$, and then disappear. At higher activity, the wave segments often give rise to rotating spiral waves. This behavior is very similar to that of chemical waves in a low excitable Belousov-Zhabotinsky (BZ) reaction. ${ }^{10}$ An example of the evolution of the spiral is shown in Figure 1. We usually observed three to five rotations of spiral waves until the surface became contaminated with a gradually forming layer of insoluble rust particles. The trajectory of the spiral tip defines an area known as the spiral wave core. ${ }^{11}$ Earlier studies on spiral waves in excitable systems revealed circular or near-circular tip trajectory. ${ }^{9-13}$ However, the trajectory of the spiral tip in the $\mathrm{Fe}-$ $\mathrm{HNO}_{3}$ system appears to be linear in general. We can see that the characteristic wavelength of the spiral is on the order of 2 $\mathrm{cm}$. This wavelength is small compared to the size of the steel 

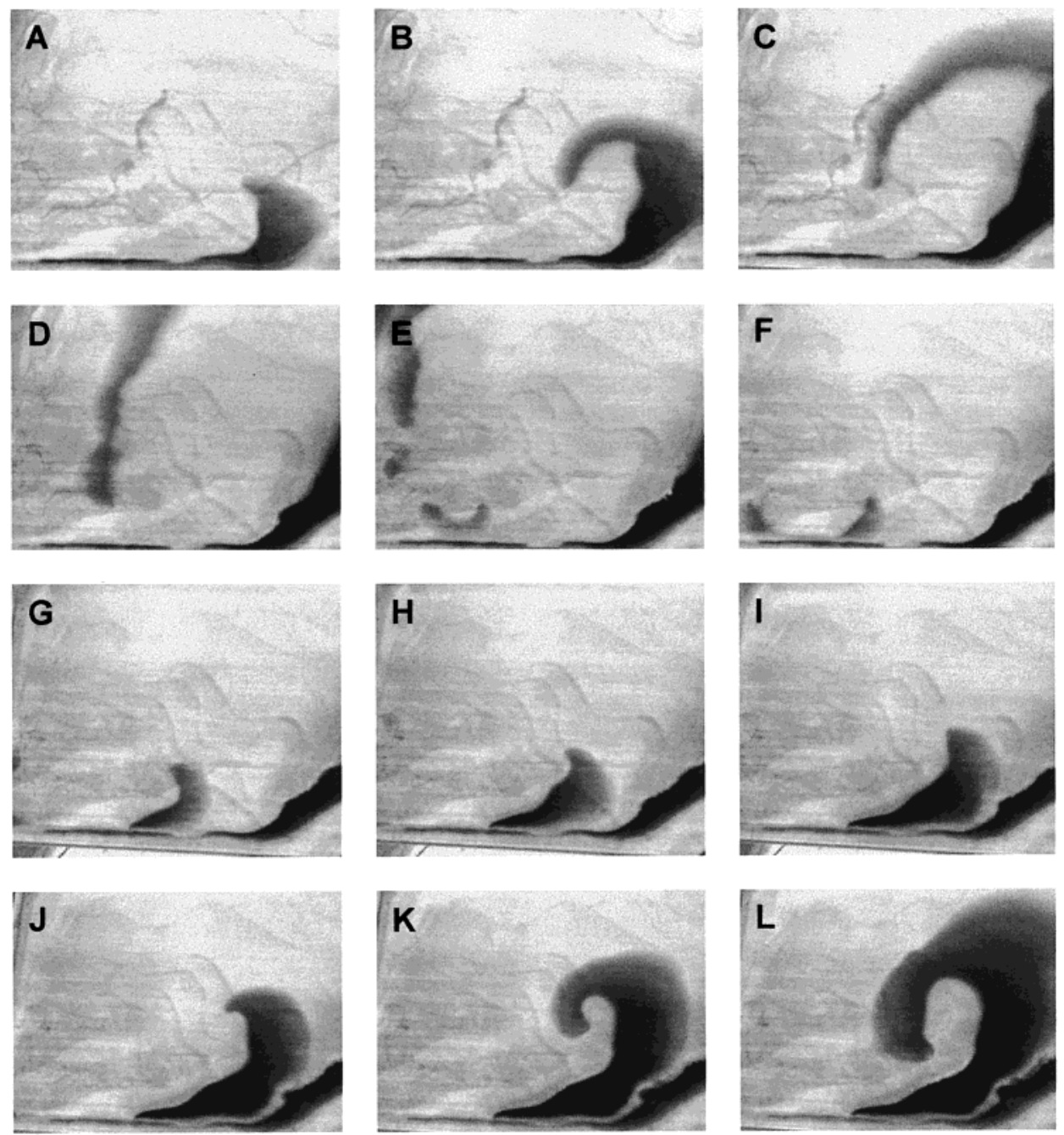

Figure 1. Propagation of waves and rotation of spirals in the iron-nitric acid system. The sequence shows 12 consecutive snapshots of the steel surface obtained at $0.7 \mathrm{~s}$ intervals. The wave is initiated at the lower left corner of the horizontal steel plate a few seconds prior to the snapshot (A). Under the given experimental conditions, the spiral waves have a rotation period of approximately $6 \mathrm{~s}$. Experimental parameters: volume ratio of nitric acid (Fisher; $15.8 \mathrm{~N}$ ) and water, 3:1 (i.e., $11.9 \mathrm{M}$ nitric acid); temperature of the solution and steel plate, $0^{\circ} \mathrm{C}$; thickness of the solution layer, $0.5 \mathrm{~mm}$; field of view, $5.9 \times 4.6 \mathrm{~cm}^{2}$. All experiments are carried out with low-carbon steel (Marshallcrat $\mathrm{C} 1018: 0.15-0.20 \% \mathrm{C}, 0.60-0.90 \%$ $\mathrm{Mn},<0.04 \% \mathrm{P},<0.05 \% \mathrm{~S}$ ) under open-circuit conditions.

plate $\left(5 \times 10 \mathrm{~cm}^{2}\right)$, and it is unlikely that the tip trajectory is influenced by the boundaries of the steel plate. ${ }^{14}$ Hence, the observed trajectory reflects the characteristic dynamics of this excitable system.

Furthermore, we observed the formation of a black trace line "drawn" by the tip of the spiral wave. A typical example for this phenomenon is presented in Figure 2. This thin, dark line shows the actual trajectory of the tip and, hence, the shape of the spiral core. After a $180^{\circ}$ turn, the spiral tip propagates in the reverse direction, often following the same black line or depositing a new line parallel to the existing one. The black traces can easily be erased by consecutive planar fronts, but they create the pathways for small wave segments and for the tips of other spiral waves. If several spiral waves coexist in the medium, the surface eventually becomes covered with chaotic black "graffiti".

Figure 3 shows two consecutive intensity profiles of a propagating wave in the $\mathrm{Fe}-\mathrm{HNO}_{3}$ system as observed along a straight line oriented perpendicular to the front. High-intensity values correspond to dark areas in the image and indicate active reaction zones. The passivated, bright areas of the system are represented by low intensities. The two wave profiles shown in Figure 3 are obtained at a $0.7 \mathrm{~s}$ interval. Contrary to what is observed most commonly in excitable media, ${ }^{1}$ the moving pulse has a steep slope in the wave back and a gradual slope in the front region. This intriguing finding is also apparent from the snapshots shown in Figure 1 and suggests that the recovery from the excited state to the excitable state might include an autocatalytic process.

The pattern formation observed in the $\mathrm{Fe}-\mathrm{HNO}_{3}$ reaction system depends significantly on the concentration of nitric acid. As mentioned above, the activity of the system increases with decreasing nitric acid concentration. In concentrated nitric acid, no patterns appear spontaneously, and external perturbations fail to nucleate waves. For a nitric acid-to-water volume ratio of $3: 1$, the reaction shows no spontaneous activity, but waves can easily be excited with a zinc rod or by local heating. With decreasing nitric acid content, localized reaction centers arise spontaneously, and the wave patterns become more complex. For a $\mathrm{HNO}_{3}$-to-water ratio lower than 3:2, we could not observe regular wave propagation at all. Under these conditions, very unstable and chaotic 

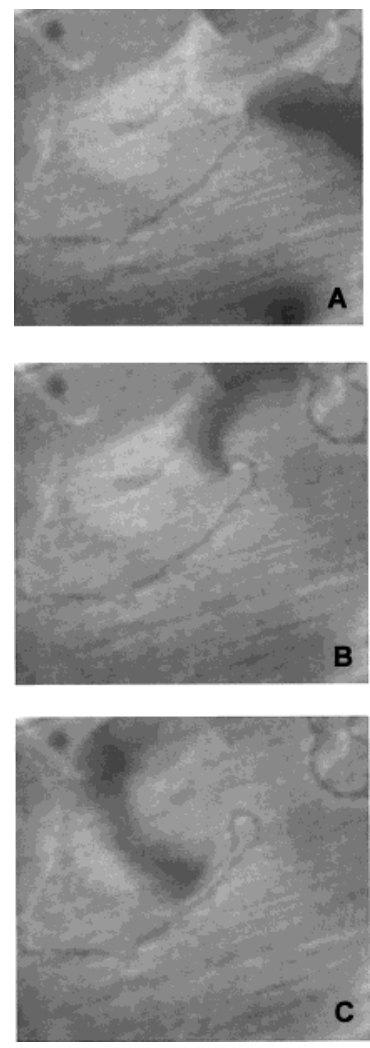

Figure 2. Spiral-tip induced formation of a hairpin-shaped black deposit that traces the tip trajectory. (A) The tip of the spiral wave moves nearly linearly from the left to the right, generating a slightly curved black line; (B) the tip of spiral waves makes a $180^{\circ}$ turn; (C) the tip propagates from the right to the left following a trajectory parallel to the black line deposited earlier. Volume ratio of nitric acid and water, 3.5:1 (i.e., $12.3 \mathrm{M}$ nitric acid); image area, $3.1 \times 4.9 \mathrm{~cm}^{2}$; time interval between snapshots, $0.7 \mathrm{~s}$.



Figure 3. Typical example of an intensity profile of a propagating wave in the $\mathrm{Fe}-\mathrm{HNO}_{3}$ system. The profiles are scanned along a line perpendicular to the wave front (time interval, $0.7 \mathrm{~s}$ ). The wave is moving from the left to the right with a constant velocity of approximately $5 \mathrm{~mm} / \mathrm{s}$. Experimental conditions as described in Figure 1.

patterns are formed, which rapidly contaminate the reaction surface with rust particles. Within this range of concentrations, we observed a transition from spiral waves to serpentine patterns, which are shown in Figure 4. In this case, small wave segments cannot curl up to rotating spirals but propagate by growing in a tangential direction while maintaining their thickness. This behavior is illustrated by the snapshots shown in Figure 4A, B. The total sequence of six snapshots reveals two individual waves which propagate from the upper right toward the lower left corner of the picture. The tail of the wave remains motionless, and propagation occurs primarily by extending the tip. Occasionally, the tip shoots out an excitation wave into the surrounding area (Figure 4C), but continuous propagation is only observed as an extension of the stationary dark tail (Figure 4D-F). With time, the tip reverses its propagation direction, and a serpentine structure is formed. Eventually, the serpentine pattern conquers the entire reaction surface, and a permanent pattern of rust bands is left behind on the steel plate.

A simplified and far from complete mechanism for the occurrence of propagating waves in the $\mathrm{Fe}-\mathrm{HNO}_{3}$ system will now be discussed. It is known that iron dissolution in concentrated nitric acid solutions can be prevented by the formation of a passivating surface film which contains considerable water of hydration..$^{7-9}$ The film is stable if the $\mathrm{pH}$ of the solution is sufficiently low. If this delicate film is damaged in some region, the process of iron dissolution resumes, thus inducing a flow of $\mathrm{Fe}^{2+}$ ions into the nearby solution layer. The local defect in the passivation film acts hereby as an anode in the forming electrolytic cell. Consequently, the positively charged layer of solution repels $\mathrm{H}^{+}$ions from the area. The local increase in $\mathrm{pH}$ destabilizes the adjacent passivation film, and additional $\mathrm{Fe}^{2+}$ is released into the solution. This process continues, and the initial defect of the passivation film propagates in a wavelike fashion. This mechanism for the propagation of the dissolution zone is similar to the dynamics of the "iron nerve" system. $^{7-9}$ The recovery process, however, appears to be different.

The recovery of the passivation film in the "iron nerve" system is due to $\mathrm{pH}$ equilibration between the bulk solution and a thin layer adjacent to the dissolving surface. This process is relatively slow and does not involve rust (i.e., $\mathrm{Fe}_{2} \mathrm{O}_{3}$ ) formation. In the experiments, however, we observe rust formation, which emphasizes the importance of dissolved oxygen as a key species in the recovery process. Consequently, oxygen also affects the whole wave dynamics. The role of oxygen was confirmed by experiments carried out under an argon atmosphere and by covering the steel surface with a glass plate. Since the solution layer has a thickness of only $0.5 \mathrm{~mm}$, dissolved $\mathrm{Fe}^{2+}$ ions can readily react with oxygen to produce rust $^{15}$

$$
4 \mathrm{Fe}^{2+}+\mathrm{O}_{2}+4 \mathrm{H}_{2} \mathrm{O}+2 \mathrm{xH}_{2} \mathrm{O} \rightarrow 2 \mathrm{Fe}_{2} \mathrm{O}_{3} \cdot x \mathrm{H}_{2} \mathrm{O}+8 \mathrm{H}^{+}
$$

This reaction apparently lowers the $\mathrm{pH}$ and, thus, favors the recovery of the passivation film. Hence, the recovery time should depend drastically on the thickness of the solution layer. Indeed, no waves could be observed when the steel plate was submerged into solutions $\geq 5 \mathrm{~mm}$ deep, regardless of the concentration of nitric acid employed.

Corrosion processes are of significant technological relevance. The corrosion patterns at later stages of the process exhibit a fascinating variety of structures. ${ }^{16,17}$ Some of them, such as filliform patterns, ${ }^{17,18}$ are apparent evidence for the existence of nontrivial mechanisms that give rise to the propagation of the active corrosion zone. We believe that the observed phenomena could be of great importance for the understanding of corrosion propagation, especially during the early stages of corrosion processes, and will have an impact on the 

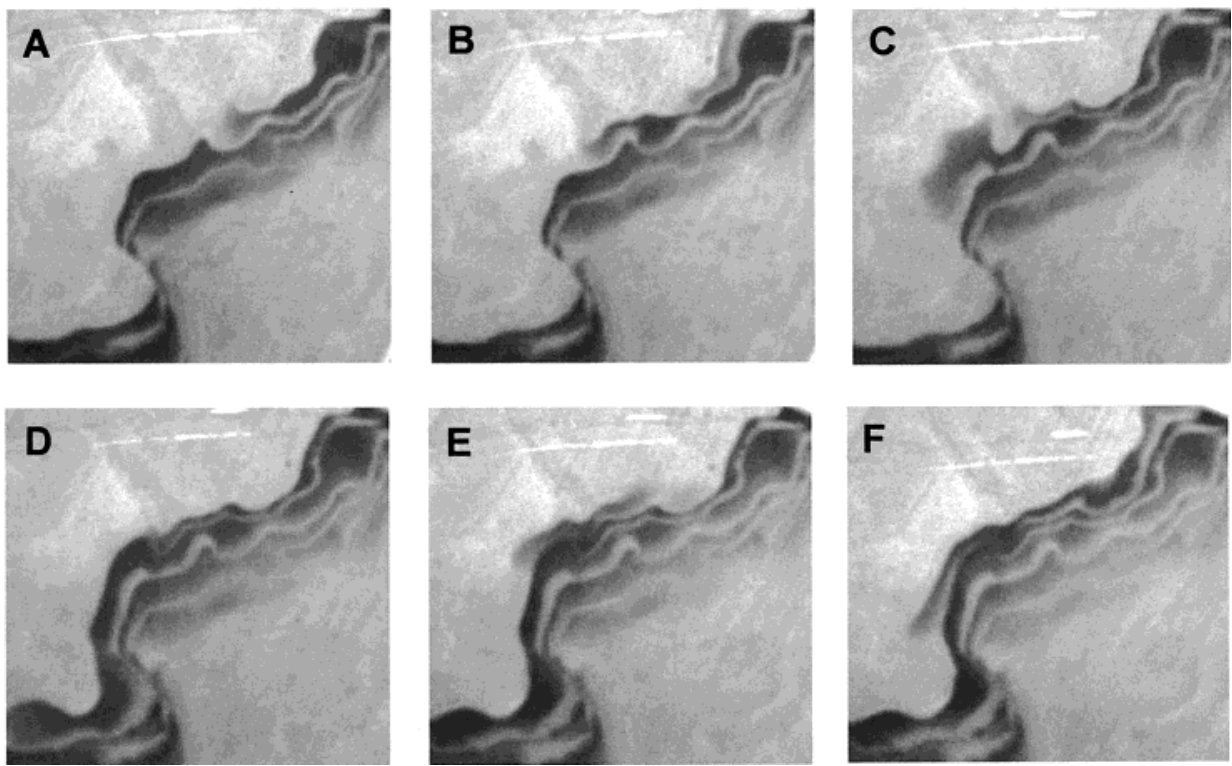

Figure 4. Serpentine motion of corrosion waves. The waves propagate from the upper right corner of the frame toward the lower left corner. Propagation occurs along the previously deposited thick S-shaped line in the central part of the frame. Experimental parameters: volume ratio of nitric acid and water, $3: 2$ (i.e., $9.5 \mathrm{M}$ nitric acid); field of view, $3.37 \times 3.62 \mathrm{~cm}^{2}$; time between snapshots, $1.4 \mathrm{~s}$; temperature, $0{ }^{\circ} \mathrm{C}$; thickness of the solution layer, $0.5 \mathrm{~mm}$.

perception of these processes in general. Furthermore, the $\mathrm{Fe}-$ $\mathrm{HNO}_{3}$ system constitutes a simple but intriguing experimental model for the investigation of chemical self-organization. Although the presented system shares some basic similarities with the well-known BZ reaction, it also opens captivating avenues for the study of new regimes of excitation propagation. A striking difference is that in the $\mathrm{Fe}-\mathrm{HNO}_{3}$ system recovery times are substantially shorter than excitation times. This feature is very unusual for excitable media and could induce exotic propagation regimes, which are predicted by theoretical studies ${ }^{19}$ but have never been observed in real systems. The list of corresponding phenomena includes echo waves, spiral waves with linear cores, reversal of spiral wave rotation, and bouncing waves. Furthermore, the serpentine wave propagation described here should provide a new impulse for the development of the theory of excitable media.

Acknowledgment. This work was supported by the Florida State University. The authors thank Stephanie ThouvenelRomans for fruitful discussions.

\section{References and Notes}

(1) Kapral, R., Showalter, K., Eds. In Chemical Waves and Patterns; Kluwer: Dordrecht, The Netherlands, 1995.

(2) Murray, J. D. Mathematical Biology; Springer: Berlin, 1989

(3) Hudson, J. L.; Tsotsis, T. T. Chem. Eng. Sci. 1994, 49, 1493.

(4) Mazouz, N.; Flatgen, G.; Krischer, K. Phys. Rev. E 1997, 55, 2260. 291.

(5) Christoph, J.; Strasser, P.; Eiswirth, M.; Ertl, G. Science 1999, 284,

(6) Lev, O.; Sheintuch, M.; Pismen, L. M.; Yarnitsky H. Nature 1988, 336,458 .

(7) Lillie, R. S. J. Gen. Physiol. 1925, 7, 473

(8) Bonhoeffer, K. F. J. Gen. Physiol. 1948, 32, 69.

(9) Suzuki, R. Adv. Biophys. 1976, 9, 115.

(10) Winfree, A. T. Prog. Theor. Chem. 1978, 4, 1.

(11) Agladze, K. I.; Krinsky, V. I. Nature 1982, 296, 424

(12) Ross, J.; Müller, S. C.; Vidal, C.; Science 1988, 240, 460.

(13) Steinbock, O.; Zykov, V.; Müller, S. C. Nature 1993, 366, 322.

(14) Sepulchre, J. A.; Babloyantz, A. Phys. Rev. E 1993, 48, 187.

(15) Sawyer, D. T.; Sobkowiak, A.; Roberts, J. L. Electrochemistry for Chemists; Wiley: New York, 1995.

(16) Van Loo, M.; Laiderman, D. D.; Bruhn R. R. Corrosion 1953, 9 , 277.

(17) Hoch, G. M. In Localized Corrosion; Staehle, R. W., Brown, B. F., Kruger, J., Agrawal A., Eds.; National Association of Corrosion Engineers: Houston, TX, 1974.

(18) Sharman, C. F. Nature 1944, 153, 621.

(19) Ermentrout, G. B.; Rinzel, J. SIAM J. Appl. Math. 1996, 56, 1107. 